\title{
Waist Circumference, Waist-to-Height Ratio and Body Mass Index as Parameters of Obesity Among Public Secondary School Students
}

\author{
Ebelechuku F. Ugochukwu, Chinyere U. Onubogu, Kenneth N. Okeke, Victoria C. Ofora, and \\ Chinenye M. Uju
}

\begin{abstract}
Background: Obesity in children is universal affecting all ethnicities, ages, gender and social classes. Public school children consist mainly of the less affluent strata of society, and consequently may not be opportuned to avail themselves of necessary public health interventions as their private school counterparts.

Objective: This study aimed to determine the prevalence of obesity using various anthropometric indices and association of certain factors with obesity among public secondary school students.
\end{abstract}

Methods: A cross-sectional school-based questionnaire and physical assessment of 593 students aged 10 - 17 years in public schools in Nnewi, Southeast Nigeria was carried out.

Results: There were 289 boys and 304 girls, giving an approximate ratio of $1: 1$. Most of the students were from the middle and lower socio-economic levels. Girls were physically less active and consumed more junk food than the boys. The mean weight and height varied significantly with age in males, females and both sexes combined together ( $p<0.001$ ). In general, girls were heavier than boys, while the boys were slightly taller than the girls. Age varied significantly with BMI ( $P<0.001$ for girls and boys), $\mathrm{WC}(\mathrm{P}<0.001$ for girls and boys) and WHtR ( $p=0.034$ for boys and 0.024 for girls). BMI performed better than WC and WHtR in identifying children with obesity. When compared with BMI, WC had a sensitivity of $5.2 \%$ and a specificity of $100.0 \%$ with a positive predictive value of $100.0 \%$ and a negative predictive value of $90.7 \%$. WHtR had a sensitivity of $31.0 \%$ and a specificity of $99.8 \%$ with a positive predictive value of $94.7 \%$ and a negative predictive value of $93.0 \%$. BMI had a strong correlation with both WC and WHtR although correlation was stronger with WC $(R=704)$ compared to WHR $(R=641)$. Among the students $9.8 \%$ were in the obese/overweight group, made up of $96.6 \%$ females, out of which $64.1 \%$ were older adolescents aged 15-17 years. Reduced physical activity played a major role in enhancing obesity/overweight, while socio-economic level and diet did not. After logistic regression analysis, age and sex remained significantly associated with being overweight or obese. The odds of being overweight or obese was 32 times in females compared to males and twice in in late adolescence compared to early adolescence.

Conclusion: The obesity/overweight of older adolescent public secondary school girls buttress the importance of physical exercises in curbing obesity. Although easier to perform and very specific, WC and WHtR seemed to miss

Published on July 23, 2020.

Ebelechuku F. Ugochukwu, Nnamdi Azikiwe University, Nigeria.

(corresponding e-mail: ef.ugochukwu@ unizik.edu.ng )

Chinyere U. Onubogu, Nnamdi Azikiwe University, Nigeria.

Kenneth N. Okeke, Nnamdi Azikiwe University, Nigeria.

Victoria C. Ofora, Nnamdi Azikiwe University Teaching Hospital, Nigeria.

Chinenye M. Uju, Nnamdi Azikiwe University Teaching Hospital, Nigeria. many adolescents with obesity when compared to BMI. Therefore, a combination of these indices is recommended while screening adolescents for obesity.

Index Terms - adolescent, anthropometric indices, obesity, public school student.

\section{INTRODUCTION}

Globally, and even surprisingly in low- and middleincome countries, particularly in urban settings, childhood obesity has been on the increase. According to the World Health Organization (WHO), it was estimated in 2016 that over 41 million infants and young children were overweight or obese, with half living in Asia and a quarter in Africa. Also 124 million children and adolescents were obese - a tenfold increase in the last four decades, giving a ratio of nearly 1 in 5 children and adolescents being overweight or obese [1].

Overweight and obese children are likely to stay obese into adulthood and more likely to develop noncommunicable diseases like diabetes and cardiovascular diseases at a younger age. Overweight and obesity have also been found to have a strong correlation with primary hypertension in children [2]-[5]. The fundamental causes behind the rising levels of childhood obesity are a shift in diet towards increased intake of energy-dense foods that are high in fat and sugars but low in vitamins, minerals and other healthy micronutrients, and more so, a trend towards decreased levels of physical activity and enhanced sedentary living.

For children and young people physical activity includes play, games, sports, chores, recreation, physical education, or planned exercise, in the context of family, school, and community activities. Children and youth should accumulate at least 60 minutes of moderate - to-vigorous-intensity physical activity daily [6]. Various methods of screening for obesity are available for field use. These include Body Mass Index (BMI), Waist Circumference (WC) and Waist-toHeight Ratio (WHtR). WC and WHtR are anthropometric indices predictive of central obesity while BMI is a measure of total body fat or adipose tissue.

Central adiposity (increased abdominal fat tissue) is associated with decreased glucose tolerance, a lowered insulin sensitivity and abnormal lipid profiles which in turn pave the way for cardiovascular diseases and type 2 diabetes. In the school health scenario it might be logistically easier to measure WC and WHtR than BMI, as tape- measuring of the waist appears easier and more likely 
reproducible than weight and height measurements (which require availability and functionality of weighing scales and stadiometers). This study therefore, compared the $\mathrm{WC}$ and WHtR to the BMI as a standard in the evaluation of the prevalence of obesity among public secondary school students; and also sought to determine factors that may be associated with adolescent obesity. The public shool students represent the regular population as private schools are usually subscribed to by the more affluent who could afford the fees.

\section{Subjects And Methods}

\section{A. Study Area and Population}

The study was conducted from September to December 2014 in Nnewi North Local Government Area (LGA) of Anambra State, South-East Nigeria. Nnewi North is a one town LGA. Most of the inhabitants are traders, civil servants or subsistence farmers, and the predominant religion is Christianity.

The study population was apparently healthy adolescent students of public secondary schools in Nnewi, aged 10 to 17 years. There were eight public secondary schools in Nnewi consisting of three all-girls' schools, two all-boys' schools and three coeducational schools with a total population of 8495 students. This population comprised of 5329 juniors and 3166 senior secondary students. The male to female ratio was 0.9: 1 which was distributed as 2500 boys and 2829 girls in the junior classes; while 1441 boys and 1725 girls were in the senior classes.

\section{B. Study Design and Subject Selection}

This was a cross-sectional descriptive study of 593 adolescent public secondary school students in Nnewi. The study participants were recruited using multi-stage stratified random sampling technique.

The schools were stratified into all-boys', all-girls', and coeducational schools. Then one school was randomly selected from each stratum. A total of three public schools were studied. Participants were proportionately selected from each school according to the school's population by multiplying the school's population with the sampling fraction (sample size/total population of selected schools). In each school, the proportional allocation was also applied to obtain the sample size for each grade. An arm was chosen from each grade by simple random sampling method. Where the allocated participants' number slightly differed from the size of the arm, the entire arm was studied. Otherwise, the students were randomly selected using the class register as sampling frame. The male: female ratio of the classes in the co-educational school was approximately 1:1 so there was no need to stratify them according to sex except where the number differed significantly from allocated number of participants.

\section{Data Collection}

Parents were notified in advance that interview as well as anthropometric measurements would be carried out on their children by a letter and a consent form. The form was to be filled, signed and returned if the parents consented to the study. In addition, assent of each participant was sought verbally prior to enrolment into the study. Where consent and assent could not be obtained, the student was not enrolled. Students who volunteered history of chronic diseases (including HIV infection, asthma, renal or cardiovascular diseases) were also excluded from the study.

An interviewer administered questionnaire was administered to each eligible student. The questionnaire captured information on date of birth, gender, class in school, family size, parental occupation and education. Physical activity, for the purposes of this study, included personal exercises - planned, structured, repetitive, strength and resistance training; school sporting activities - track and field games; cycling, skipping, brisk walking, dancing, soccer, basketball, etc. The more vigorous activities performed at least for an hour every day of the week, were categorized as intensive. Moderate activities like brisk walking, stationary exercises, table tennis carried out for about an hour three to five days a week constituted moderate physical activity. Occasional walks with a frequency less than three days a week, and duration not up to an hour was classified as minimal physical activity. Physical activity was assessed by asking about the type of sports and games the students were regularly involved in, as well as the frequency and duration of activities on a weekly basis. A 24-hour dietary recall of all foods and drinks consumed was also documented for each student. Fizzy drinks, soda, high energy fried and baked pastries were regarded as junk food. Regular staple meals consisted of a good blend of the food classes with vegetables and fruits.

The second phase of the study involved physical examination of the students and measurement of height, weight and waist circumference. The students were examined in their school uniforms without cardigans, shoes and socks. Weights of the students were measured using a ZT- 120 Health scale, to the nearest $0.5 \mathrm{~kg}$.

The height was measured with a ZT-120 Stadiometer that had a moveable headpiece perpendicular to the vertical backboard. The student stood with the heels, buttocks, shoulders and head touching the vertical backboard, the neck slightly stretched and eyes looking directly ahead. The headpiece was gently brought down until it compressed the hair without exerting any pressure on the head. The measurement was read with the eyes directly facing the level of the headboard in the horizontal plane and was recorded to the nearest 0.5 centimeters.

The waist circumference was obtained at the end of several consecutive natural breaths, at a level parallel to the floor, midpoint between the lower margin of the last palpable rib and the top of the iliac crest in the mid axillary line [7]. A stretch-resistant tape was snugly wrapped around the abdomen in a non-constricting manner. The tape was kept level and parallel to the floor at the point of measurement with the subject standing upright, arms relaxed at the side and feet evenly spread apart, allowing even distribution of the body weight.

\section{Data Management and Analysis}

Data was analyzed using Statistical Package for Social Sciences (SPSS) (IBM, Chicago IL, USA) software version 20. The mean and standard deviation for continuous variables such as BMI, WC and WHtR were calculated for different age and sex in univariate analysis. Percentiles 
charts for BMI, WC and WHtR were also generated for age and sex. The relationship between categorical and continuous variables was examined using Pearson's Chi square test. Where assumptions for Chi-square test was violated, Fisher's exact test was used. For each sex, the mean BMI, WC or WHtR was compared across different age categories using one-way ANOVA while Pearson's correlation was used to compare relationship between continuous variables. Factors significantly associated with overweight/obesity were further analyzed using binary logistic regression analysis. Level of significance was set at $\mathrm{p}<0.05$ and $95 \%$ confidence interval.

WHO percentile and z-score charts for BMI for age and sex [8] and the Children's Hospital Research Institute of Manitoba, Canada, WHR and WC percentile charts [9] were used as standard for comparison. Body mass index was calculated as weight $(\mathrm{kg})$ divided by the square of the height (metres) and reported as $\mathrm{kg} / \mathrm{m}^{2}$. BMI of $>+2 \mathrm{SD}$ was considered obese, $>+1 \mathrm{SD}$ (overweight), $\leq+1 \mathrm{SD}$ to $>-2 \mathrm{SD}$ (normal or healthy), <-2SD (thinness), and <-3SD (severe thinness). WHtR was calculated as WC in centimetres $(\mathrm{cm})$ divided by height in $\mathrm{cm}$. Values of $\leq 0.5$ were regarded as normal, $>0.5$ but $<0.6$ (overweight) and $\geq 0.6$ as obesity. WC $\geq 90$ th percentile was categorized as central obesity, while values $<90$ th percentile were normal.

Socioeconomic level was worked out using a modification of Oyedeji's classification [10].

\section{RESUltS}

Out of the 593 students recruited in the study 289 were boys and 304 girls, giving an approximate ratio of 1:1. Most of the students were from the middle and lower socioeconomic levels. Girls were physically less active and consumed more junk food than the boys. The characteristics of the students studied are outlined in Table I.

The mean weight and height varied significantly with age in males, females and both sexes combined together ( $p$ $<0.001$ for all). In general, girls were heavier than boys, while the boys were slightly taller than the girls as shown in Table II. Age had a strong correlation with both weight (0.559) and height (0.673).

TABLE I. SOCIO-DEMOGRAPHIC CHARACTERISTICS OF SUBJECTS

\begin{tabular}{|c|c|c|c|c|}
\hline \multirow{2}{*}{\multicolumn{2}{|c|}{ Characteristic }} & \multicolumn{2}{|c|}{ Gender } & \multirow{3}{*}{$\begin{array}{l}\text { Total } \\
3(0.5) \\
\end{array}$} \\
\hline & & \multirow{2}{*}{$\begin{array}{l}\text { Males } \\
1(0.3)\end{array}$} & \multirow{2}{*}{$\begin{array}{c}\text { Females } \\
2(0.7)\end{array}$} & \\
\hline \multirow{9}{*}{$\begin{array}{c}\text { Age } \\
\text { (years) }\end{array}$} & 10 & & & \\
\hline & 11 & $1(0.3)$ & $11(3.6)$ & $12(2.0)$ \\
\hline & 12 & $12(4.2)$ & $47(15.5)$ & $59(9.9)$ \\
\hline & 13 & $67(23.2)$ & $49(16.1)$ & $116(19.6)$ \\
\hline & 14 & $80(27.7)$ & $42(13.8)$ & $122(20.6)$ \\
\hline & 15 & $58(20.1)$ & $66(21.7)$ & $124(20.9)$ \\
\hline & 16 & $50(17.3)$ & $52(17.1)$ & $102(17.2)$ \\
\hline & 17 & $20(6.9)$ & $35(11.5)$ & $55(9.3)$ \\
\hline & Total & $289(100.0)$ & $304(100.0)$ & $593(100.0)$ \\
\hline \multirow{3}{*}{$\begin{array}{l}\text { Class in } \\
\text { School }\end{array}$} & Junior Sec & $193(66.8)$ & $169(55.6)$ & $362(61.0)$ \\
\hline & Senior Sec & $96(33.2)$ & $135(44.4)$ & $231(39.0)$ \\
\hline & Total & $289(100.0)$ & $304(100.0)$ & $593(100.0)$ \\
\hline \multirow{4}{*}{$\begin{array}{c}\text { Socioeco } \\
\text { nomic } \\
\text { Level }\end{array}$} & Upper & $15(5.2)$ & $13(4.3)$ & $28(4.7)$ \\
\hline & Middle & $104(36.0)$ & $114(37.5)$ & $218(36.8)$ \\
\hline & Lower & $170(58.8)$ & $177(58.2)$ & $347(58.5)$ \\
\hline & Total & $289(100.0)$ & $304(100.0)$ & $593(100.0)$ \\
\hline \multirow{4}{*}{$\begin{array}{l}\text { Physical } \\
\text { Activity }\end{array}$} & Minimal & $150(51.9)$ & $272(89.5)$ & $422(71.2)$ \\
\hline & Moderate & $88(30.4)$ & $28(9.2)$ & $116(19.6)$ \\
\hline & Intensive & $51(17.6)$ & $4(1.3)$ & $55(9.3)$ \\
\hline & Total & $289(100.0)$ & $304(100.0)$ & $593(100.0)$ \\
\hline \multirow{4}{*}{ Diet } & $\begin{array}{c}\text { Regular } \\
\text { staple meals }\end{array}$ & $233(80.9)$ & $180(59.8)$ & $413(70.1)$ \\
\hline & $\begin{array}{c}\text { Regular } \\
\text { staple meals }+ \\
\text { junk foods }\end{array}$ & $12(4.2)$ & $46(15.3)$ & $58(9.8)$ \\
\hline & $\begin{array}{l}\text { Junk foods } \\
\text { replacing one } \\
\text { or more meals }\end{array}$ & $43(14.9)$ & 75 (24.9) & $118(20.0)$ \\
\hline & Total & $288(100.0)$ & $301(100.0)$ & $589(100.0)$ \\
\hline
\end{tabular}

Percentages are in parentheses.

TABLE II. MEAN AND STANDARD DEVIATION OF ANTHROPOMETRIC INDICES OF STUDENTS ACCORDING TO AGE AND SEX

\begin{tabular}{|c|c|c|c|c|c|c|c|c|c|c|c|}
\hline \multirow{2}{*}{$\begin{array}{c}\text { Age } \\
\text { (years) }\end{array}$} & \multicolumn{2}{|c|}{ Weight (kg) } & \multicolumn{2}{|c|}{ Height $(\mathrm{cm})$} & \multicolumn{2}{|c|}{ BMI $\left(\mathrm{kg} / \mathrm{m}^{2}\right)$} & \multicolumn{2}{|c|}{$\mathrm{WC}(\mathrm{cm})$} & \multicolumn{2}{|c|}{ WHtR } & \multirow[t]{2}{*}{$\mathrm{N}$} \\
\hline & Mean & SD & Mean & SD & Mean & SD & Mean & SD & Mean & SD & \\
\hline Males & & & & & & & & & & & \\
\hline 10 & 35.5 & - & 141.0 & - & 17.9 & - & 60.0 & - & 0.43 & - & 1 \\
\hline 11 & 33.0 & - & 145.0 & - & 15.7 & - & 56.8 & - & 0.39 & - & 1 \\
\hline 12 & 36.8 & 4.71 & 146.2 & 6.44 & 17.2 & 1.5 & 61.8 & 3.7 & 0.42 & 0.01 & 12 \\
\hline 13 & 39.5 & 6.18 & 151.4 & 7.98 & 17.2 & 1.7 & 63.9 & 4.6 & 0.42 & 0.02 & 67 \\
\hline 14 & 43.3 & 6.74 & 155.8 & 8.29 & 17.8 & 1.7 & 64.9 & 4.2 & 0.42 & 0.03 & 80 \\
\hline 15 & 49.1 & 8.35 & 162.7 & 8.92 & 18.4 & 1.9 & 67.2 & 4.7 & 0.41 & 0.03 & 58 \\
\hline 16 & 52.9 & 7.53 & 167.8 & 7.72 & 18.7 & 1.9 & 68.6 & 4.1 & 0.41 & 0.03 & 50 \\
\hline 17 & 58.0 & 8.23 & 169.5 & 6.86 & 20.2 & 2.5 & 70.7 & 3.5 & 0.41 & 0.02 & 20 \\
\hline Total & 45.9 & 9.29 & 158.7 & 10.61 & 18.1 & 2.0 & 66.0 & 4.9 & 0.42 & 0.03 & 289 \\
\hline Females & & & & & & & & & & & \\
\hline 10 & 34.5 & 3.54 & 146.8 & 3.89 & 16.0 & 0.8 & 58.2 & 2.3 & 0.40 & 0.01 & 2 \\
\hline 11 & 38.3 & 6.22 & 146.2 & 6.59 & 17.9 & 2.7 & 62.5 & 4.1 & 0.43 & 0.03 & 11 \\
\hline 12 & 42.7 & 8.50 & 152.5 & 9.36 & 18.5 & 2.9 & 64.7 & 5.8 & 0.43 & 0.03 & 47 \\
\hline 13 & 47.5 & 8.46 & 154.9 & 7.02 & 19.7 & 3.1 & 67.8 & 6.2 & 0.44 & 0.04 & 49 \\
\hline 14 & 50.0 & 7.57 & 156.0 & 6.23 & 20.5 & 2.6 & 68.3 & 4.7 & 0.44 & 0.03 & 42 \\
\hline 15 & 56.9 & 9.19 & 158.5 & 5.43 & 22.6 & 3.3 & 70.8 & 6.8 & 0.45 & 0.04 & 66 \\
\hline 16 & 56.1 & 7.58 & 159.8 & 7.44 & 22.1 & 2.6 & 68.1 & 4.8 & 0.43 & 0.03 & 52 \\
\hline 17 & 56.3 & 6.96 & 159.4 & 5.99 & 22.1 & 2.5 & 68.6 & 5.9 & 0.43 & 0.04 & 35 \\
\hline Total & 51.2 & 10.02 & 156.4 & 7.65 & 20.9 & 3.3 & 67.9 & 6.1 & 0.44 & 0.04 & 304 \\
\hline Both & & & & & & & & & & & \\
\hline 10 & 34.8 & 2.57 & 34.8 & 4.31 & 16.6 & 1.2 & 58.8 & 2.0 & 0.41 & 0.02 & 3 \\
\hline 11 & 37.9 & 6.13 & 37.9 & 6.30 & 17.7 & 2.6 & 62.1 & 4.2 & 0.42 & 0.03 & 12 \\
\hline 12 & 41.5 & 8.18 & 41.5 & 9.15 & 18.2 & 2.7 & 64.1 & 5.5 & 0.43 & 0.03 & 59 \\
\hline 13 & 42.9 & 8.21 & 42.9 & 7.75 & 18.3 & 2.7 & 65.6 & 5.6 & 0.43 & 0.03 & 116 \\
\hline 14 & 45.6 & 7.71 & 45.6 & 7.62 & 18.7 & 2.4 & 66.1 & 4.7 & 0.42 & 0.03 & 122 \\
\hline 15 & 53.3 & 9.59 & 53.3 & 7.55 & 20.7 & 3.4 & 69.1 & 6.2 & 0.43 & 0.04 & 124 \\
\hline 16 & 54.5 & 7.69 & 54.5 & 8.54 & 20.4 & 2.8 & 68.3 & 4.5 & 0.42 & 0.03 & 102 \\
\hline 17 & 56.9 & 7.41 & 56.9 & 7.93 & 21.4 & 2.6 & 69.4 & 5.2 & 0.43 & 0.03 & 55 \\
\hline Total & 48.6 & 10.02 & 48.6 & 9.28 & 19.5 & 3.1 & 67.0 & 5.6 & 0.43 & 0.03 & 593 \\
\hline
\end{tabular}


In males, age varied significantly with $\mathrm{BMI}(\mathrm{P}<0.001)$, WC $(\mathrm{P}<0.001)$ and $\mathrm{WHtR}(0.034)$. Likewise, in females, age varied significantly with BMI $(\mathrm{P}<0.001)$, WC $(\mathrm{P}<0.001)$ and WHtR $(\mathrm{p}=0.024)$.

BMI performed better in identifying children with obesity. When compared with BMI, WC had a sensitivity of $5.2 \%$ and a specificity of $100.0 \%$ with a positive predictive value of $100.0 \%$ and a negative predictive value of $90.7 \%$.
WHtR had a sensitivity of $31.0 \%$ and a specificity of $99.8 \%$ with a positive predictive value of $94.7 \%$ and a negative predictive value of $93.0 \%$.

BMI had a strong correlation with both WC and WHtR although correlation was stronger with WC $(\mathrm{R}=704)$ compared to WHR (R=641). Tables III and IV outline the anthropometric parameters in their various percentile ranges according to ages for both sexes.

TABLE III. PERCENTILE VALUES OF ANTHROPOMETRIC INDICES FOR GIRLS

\begin{tabular}{|c|c|c|c|c|c|c|c|c|c|}
\hline \multirow{2}{*}{ PARAMETER } & \multirow{2}{*}{ Age (years } & \multicolumn{7}{|c|}{ PERCENTILES FOR GIRLS } & \multirow[t]{2}{*}{$\begin{array}{c}\mathrm{N} \\
(304) \\
\end{array}$} \\
\hline & & 5 & 10 & 25 & 50 & 75 & 90 & 95 & \\
\hline \multirow{8}{*}{ BMI $\left(\mathrm{kg} / \mathrm{m}^{2}\right)$} & 10 & - & - & - & 16.0 & - & - & - & 2 \\
\hline & 11 & 14.3 & 14.5 & 15.6 & 18.2 & 20.9 & 21.5 & - & 11 \\
\hline & 12 & 14.8 & 15.5 & 16.4 & 17.4 & 20.4 & 24.3 & 24.5 & 47 \\
\hline & 13 & 15.7 & 16.1 & 17.4 & 19.6 & 21.1 & 22.8 & 25.1 & 49 \\
\hline & 14 & 16.5 & 17.6 & 19.2 & 19.9 & 21.4 & 24.1 & 25.2 & 42 \\
\hline & 15 & 17.4 & 18.7 & 20.0 & 21.9 & 24.6 & 28.0 & 28.7 & 66 \\
\hline & 16 & 18.4 & 18.7 & 20.2 & 21.9 & 24.1 & 26.3 & 27.1 & 52 \\
\hline & 17 & 18.9 & 19.9 & 20.5 & 21.7 & 23.4 & 25.0 & 27.4 & 35 \\
\hline \multirow{8}{*}{$\mathrm{WC}(\mathrm{cm})$} & 10 & - & - & 56.5 & 58.2 & - & - & & 2 \\
\hline & 11 & 56.0 & 56.2 & 59.0 & 64.0 & 66.4 & 67.4 & & 11 \\
\hline & 12 & 56.7 & 57.8 & 60.8 & 63.4 & 69.4 & 72.6 & 76.8 & 47 \\
\hline & 13 & 57.8 & 60.0 & 63.0 & 67.5 & 72.0 & 75.0 & 76.0 & 49 \\
\hline & 14 & 61.0 & 61.3 & 64.7 & 68.4 & 71.5 & 73.5 & 77.0 & 42 \\
\hline & 15 & 61.0 & 63.0 & 66.3 & 69.0 & 74.0 & 80.3 & 85.9 & 66 \\
\hline & 16 & 61.7 & 62.2 & 63.6 & 68.0 & 70.6 & 75.0 & 78.4 & 52 \\
\hline & 17 & 59.0 & 61.1 & 65.0 & 68.5 & 72.0 & 74.3 & 79.4 & 35 \\
\hline \multirow{8}{*}{ WHtR } & 10 & - & - & 0.39 & 0.40 & & & & 2 \\
\hline & 11 & 0.38 & 0.38 & 0.40 & 0.43 & 0.46 & 0.46 & & 11 \\
\hline & 12 & 0.38 & 0.38 & 0.40 & 0.42 & 0.44 & 0.48 & 0.49 & 47 \\
\hline & 13 & 0.39 & 0.39 & 0.41 & 0.44 & 0.46 & 0.48 & 0.50 & 49 \\
\hline & 14 & 0.39 & 0.40 & 0.42 & 0.43 & 0.46 & 0.47 & 0.49 & 42 \\
\hline & 15 & 0.37 & 0.40 & 0.42 & 0.45 & 0.47 & 0.50 & 0.53 & 66 \\
\hline & 16 & 0.39 & 0.39 & 0.40 & 0.42 & 0.45 & 0.47 & 0.48 & 52 \\
\hline & 17 & 0.37 & 0.39 & 0.41 & 0.43 & 0.46 & 0.47 & 0.49 & 35 \\
\hline
\end{tabular}

TABLE IV. PERCENTILE VALUES OF ANTHROPOMETRIC INDICES FOR BOYS

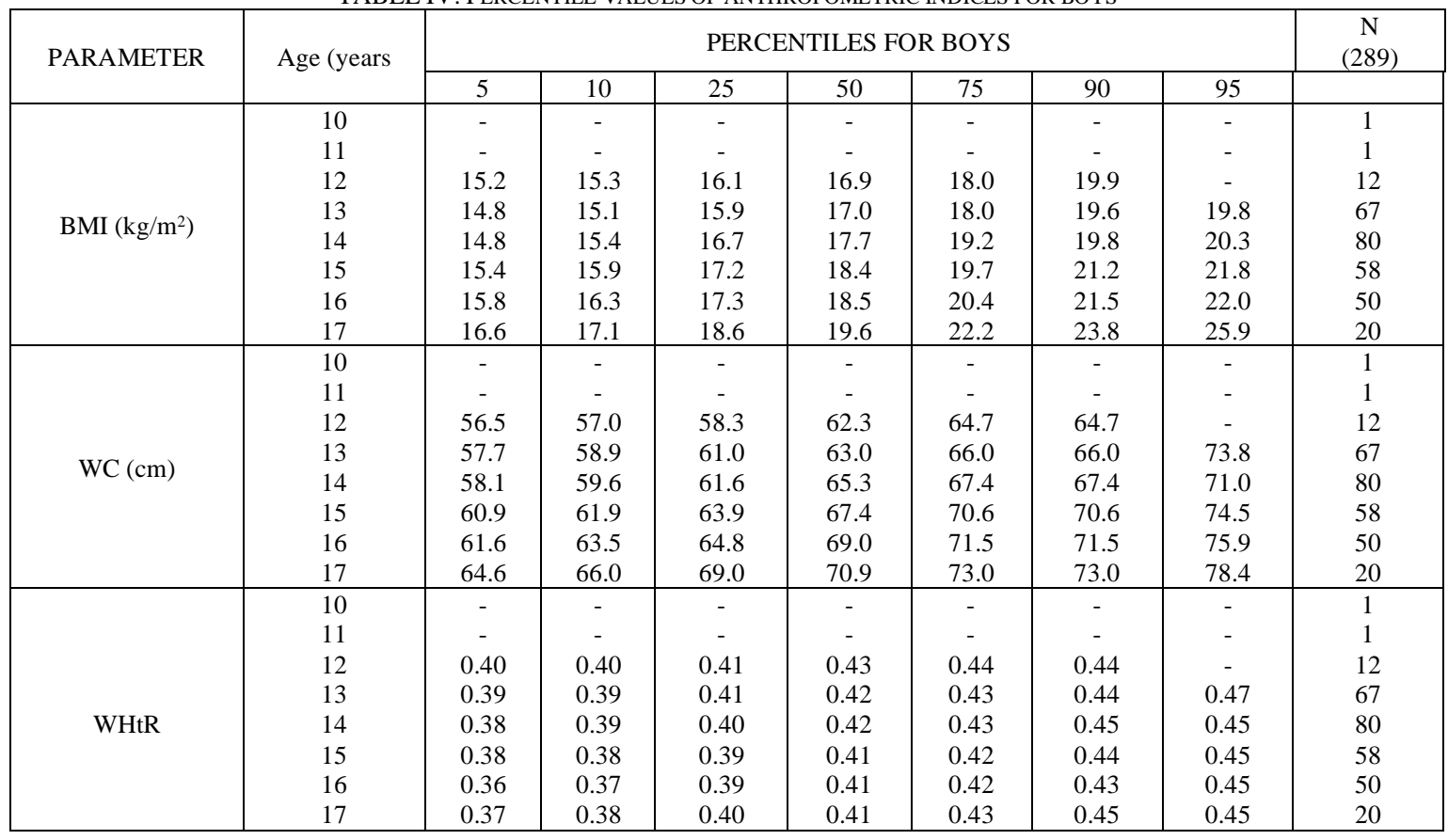

Table $\mathrm{V}$ shows that all the indices used in assessing obesity/overweight were higher in girls than boys for global obesity (BMI) and for central obesity (WC and WHtR). It is noteworthy that $7.3 \%$ of the students were underweight $18.6 \%$ of them were girls (all aged 10-14 years), while $81.4 \%$ were boys $(42.9 \%$ younger and $57.1 \%$ older adolescents).
Table VI highlights that obesity and overweight were found more in girls than boys. Older adolescent girls were significantly more obese than their younger counterparts. Among the students $9.8 \%$ were in the obese/overweight group, made up of $96.6 \%$ females, out of which $64.1 \%$ were older adolescents aged 15-17 years. Reduced physical activity also played a major role in enhancing 
obesity/overweight, while socio-economic level and diet did

not.

TABLE V. NUTRITIONAL STATUS CLASSIFICATION BY ANTHROPOMETRIC INDICES

\begin{tabular}{|c|c|c|c|c|}
\hline \multirow[t]{2}{*}{ Age } & $\begin{array}{l}\text { Anthropometric } \\
\text { Index }\end{array}$ & Males & Females & Total \\
\hline & \multicolumn{4}{|c|}{ BMI Percentile Category } \\
\hline \multirow{6}{*}{$10-14$ years } & Obesity [>+2SD] & $0(0.0)$ & $2(1.3)$ & $2(0.6)$ \\
\hline & Overweight [>+1SD] & $2(1.2)$ & 18(11.9) & $20(6.4)$ \\
\hline & Normal/Healthy $[\leq+1 \mathrm{SD}$ to $>-2 \mathrm{SD}]$ & $144(89.4)$ & $123(81.5)$ & $267(85.6)$ \\
\hline & Thinness [<-2SD] & $13(8.1)$ & $8(5.3)$ & $21(6.7)$ \\
\hline & Severe thinness [ $<-3 \mathrm{SD}]$ & $2(1.2)$ & $0(0.0)$ & $2(0.6)$ \\
\hline & Total & $161(100.0)$ & $151(100.0)$ & $312(100.0)$ \\
\hline \multirow{6}{*}{$15-17$ years } & Obesity [>+2SD] & $0(0.0)$ & $4(2.6)$ & $4(1.4)$ \\
\hline & Overweight [>+1SD] & $0(0.0)$ & $32(20.9)$ & $32(11.4)$ \\
\hline & Normal/Healthy $[\leq+1 \mathrm{SD}$ to $>-2 \mathrm{SD}]$ & $108(84.4)$ & $117(76.5)$ & $225(80.1)$ \\
\hline & Thinness [<-2SD] & $19(14.8)$ & $0(0.0)$ & $19(6.8)$ \\
\hline & Severe thinness $[<-3 S D]$ & $1(0.8)$ & $0(0.0)$ & $1(0.4)$ \\
\hline & Total & $128(100.0)$ & $153(100.0)$ & $281(100.0)$ \\
\hline \multirow{6}{*}{ All } & Obesity [>+2SD] & $0(0.0)$ & $6(2.0)$ & $6(1.0)$ \\
\hline & Overweight [>+1SD] & $2(0.7)$ & $50(16.4)$ & $52(8.8)$ \\
\hline & Normal/Healthy $[\leq+1 \mathrm{SD}$ to $>-2 \mathrm{SD}]$ & $252(87.2)$ & $240(78.9)$ & $492(83.0)$ \\
\hline & Thinness [<-2SD] & $32(11.1)$ & $8(2.6)$ & $40(6.7)$ \\
\hline & Severe thinness $[<-3 \mathrm{SD}]$ & $3(1.0)$ & $0(0.0)$ & $3(0.5)$ \\
\hline & Total & $289(100.0)$ & $304(100.0)$ & $593(100.0)$ \\
\hline \multirow{5}{*}{$10-14$ years } & Wais & Ratio Catego & & \\
\hline & Obesity $[\geq 0.6]$ & $0(0.0)$ & $1(0.7)$ & $1(0.3)$ \\
\hline & Overweight $[0.5$ to $<0.6]$ & $2(1.2)$ & $4(2.6)$ & $6(1.9)$ \\
\hline & Normal $[<0.5]$ & $159(98.8)$ & $146(96.7)$ & $305(97.8)$ \\
\hline & Total & $161(100.0)$ & $151(100.0)$ & $312(100.0)$ \\
\hline \multirow{4}{*}{$15-17$ years } & Obesity $[\geq 0.6]$ & $0(0.0)$ & $0(0.0)$ & $0(0.0)$ \\
\hline & Overweight $[0.5$ to $<0.6]$ & $1(0.8)$ & $11(7.2)$ & $12(4.3)$ \\
\hline & Normal $[<0.5]$ & $127(99.2)$ & $142(92.8)$ & $269(95.7)$ \\
\hline & Total & $128(100.0)$ & $153(100.0)$ & $281(100.0)$ \\
\hline \multirow{4}{*}{ All } & Obesity $[\geq 0.6]$ & $0(0.0)$ & $1(0.3)$ & $1(0.2)$ \\
\hline & Overweight $[0.5$ to $<0.6]$ & $3(1.0)$ & $15(4.9)$ & $18(3.0)$ \\
\hline & Normal $[<0.5]$ & $286(99.0)$ & $288(94.7)$ & $574(96.8)$ \\
\hline & Total & $289(100.0)$ & $304(100.0)$ & $593(100.0)$ \\
\hline \multirow{4}{*}{$10-14$ years } & Wais & ence Catego & & \\
\hline & Central Obesity [ $\geq 90^{\text {th }}$ percentile] & $0(0.0)$ & $2(1.3)$ & $2(0.6)$ \\
\hline & Normal $\left[<90^{\text {th }}\right.$ percentile] & $161(100.0)$ & $149(98.7)$ & $310(99.4)$ \\
\hline & Total & $161(100.0)$ & $151(100.0)$ & $312(100.0)$ \\
\hline \multirow{3}{*}{$15-17$ years } & Central Obesity [ $\geq 90^{\text {th }}$ percentile] & $0(0.0)$ & $1(0.7)$ & $1(0.4)$ \\
\hline & Normal $\left[<90^{\text {th }}\right.$ percentile $]$ & $128(100.0)$ & $152(99.3)$ & $280(99.6)$ \\
\hline & Total & $128(100.0)$ & $153(100.0)$ & $281(100.0)$ \\
\hline \multirow{3}{*}{ All } & Central Obesity [ $\geq 90^{\text {th }}$ percentile $]$ & $0(0.0)$ & $3(1.0)$ & $3(0.5)$ \\
\hline & Normal $\left[<90^{\text {th }}\right.$ percentile $]$ & $289(100.0)$ & $301(100.0)$ & $590(99.5)$ \\
\hline & Total & $289(100.0)$ & $304(100.0)$ & $593(100.0)$ \\
\hline
\end{tabular}

Percentages in parentheses.

TABLE VI. FACTORS ASSOCIATED WITH OBESITY

\begin{tabular}{|c|c|c|c|c|c|}
\hline \multirow{2}{*}{\multicolumn{2}{|c|}{ Variables }} & \multicolumn{2}{|c|}{ BMI Categories } & \multirow[b]{2}{*}{ Total } & \multirow[b]{2}{*}{ P-value } \\
\hline & & Obese/ & Normal/ & & \\
\hline \multirow{3}{*}{ Sex } & Boys & $2(0.7)$ & $287(99.3)$ & $289(100.0)$ & \multirow{3}{*}{$<0.001^{* *}$} \\
\hline & Girls & $56(18.4)$ & $248(81.6)$ & $304(100.0)$ & \\
\hline & Total & $58(9.8)$ & $535(90.2)$ & $593(100.0)$ & \\
\hline \multirow{2}{*}{$\begin{array}{c}\begin{array}{c}\text { Age for Boys } \\
\text { (years) }\end{array} \\
\end{array}$} & $10-14$ & $2(1.2)$ & $159(98.8)$ & $161(100.0)$ & \multirow{2}{*}{$0.505^{*}$} \\
\hline & $15-17$ & $0(0.0)$ & $128(100.0)$ & $128(100.0)$ & \\
\hline \multirow{2}{*}{$\begin{array}{c}\text { Age for Girls } \\
\text { (years) }\end{array}$} & $10-14$ & $20(13.2)$ & $131(86.8)$ & $151(100.0)$ & \multirow{2}{*}{$0.021^{* *}$} \\
\hline & $15-17$ & $36(23.5)$ & $117(76.5)$ & $153(100.0)$ & \\
\hline \multirow{3}{*}{ Age for Both Sexes (years) } & $10-14$ & $22(7.1)$ & $290(92.9)$ & $312(100.0)$ & \multirow{3}{*}{$0.018^{* *}$} \\
\hline & $15-17$ & $36(12.8)$ & $245(87.2)$ & $281(100.0)$ & \\
\hline & Total & $58(9.8)$ & $535(90.2)$ & $593(100.0)$ & \\
\hline \multirow{4}{*}{ Diet } & Regular staple meals & $34(8.2)$ & $379(91.8)$ & $413(100.0)$ & \multirow{4}{*}{$0.187^{* *}$} \\
\hline & Regular staple meals plus snacking junk foods & $8(13.8)$ & $50(86.2)$ & $58(100.0)$ & \\
\hline & Junk foods replacing one or more meals & $15(12.7)$ & $103(87.3)$ & $118(100.0)$ & \\
\hline & Total & $57(9.7)$ & $532(90.3)$ & $589(100.0)$ & \\
\hline \multirow{4}{*}{$\begin{array}{c}\text { Socioeconomic } \\
\text { level }\end{array}$} & Upper & $2(7.1)$ & $26(92.9)$ & $28(100.0)$ & \multirow{4}{*}{$0.820^{* *}$} \\
\hline & Middle & $23(10.6)$ & $195(89.4)$ & $218(100.0)$ & \\
\hline & Lower & $33(9.5)$ & $314(90.5)$ & $347(100.0)$ & \\
\hline & Total & $58(9.8)$ & $535(90.2)$ & $593(100.0)$ & \\
\hline \multirow{4}{*}{ Physical Activity } & Minimal & $51(12.1)$ & $371(87.9)$ & $422(100.0)$ & \multirow{4}{*}{$0.006^{* *}$} \\
\hline & Moderate & $7(6.0)$ & $109(94.0)$ & $116(100.0)$ & \\
\hline & Intense & $0(0.0)$ & $55(100.0)$ & $55(100.0)$ & \\
\hline & Total & $58(9.8)$ & $535(90.2)$ & $593(100.0)$ & \\
\hline
\end{tabular}

Percentages in parentheses. *Fisher's Exact test. **Pearson Chi-square test. 
After logistic regression analysis, age and sex remained significantly associated with being overweight or obese. The odds of being overweight or obese is 32 times in females compared to males and twice in in late adolescence compared to early adolescence as shown in Table VII.

TABLE VII. LOGISTIC REGRESSION ANALYSIS FOR FACTORS SIGNIFICANTLY ASSOCIATED WITH OBESITY/OVERWEIGHT

\begin{tabular}{|c|c|c|c|c|c|c|}
\hline \multirow{2}{*}{ Parameter } & \multicolumn{4}{|c|}{ Odds ratio estimates } & \multicolumn{2}{c|}{ Risk estimates } \\
\cline { 2 - 7 } & df & OR & $95 \% \mathrm{C} 1$ & $\begin{array}{c}\text { p- } \\
\text { value }\end{array}$ & RR & $95 \% \mathrm{CI}$ \\
\hline $\begin{array}{c}\text { Sex: Female vs } \\
\text { Males }\end{array}$ & 1 & 31.22 & $\begin{array}{c}7.25- \\
134.44\end{array}$ & $<0.001$ & 26.62 & $\begin{array}{c}6.56- \\
108.07\end{array}$ \\
\hline Age : & 1 & 1.81 & $\begin{array}{c}1.01- \\
3.25\end{array}$ & 0.046 & 1.81 & $\begin{array}{c}1.10- \\
3.01\end{array}$ \\
$\begin{array}{c}\text { 15-17years vs 10- } \\
\text { 14years }\end{array}$ & 1 & 0.96 & $\begin{array}{c}0.39- \\
2.34\end{array}$ & 0.922 & 2.95 & $\begin{array}{c}1.37- \\
6.38\end{array}$ \\
\hline $\begin{array}{c}\text { Sporting activity: } \\
\text { Minimal vs } \\
\text { Moderate/intense }\end{array}$ & 1 & & & & & \\
\hline
\end{tabular}

\section{DISCUSSION}

There has been a gradual trend towards overweight and obesity in youths all over the world, developing countries inclusive. Several interventional measures have also been advanced to curb this trend to mitigate associated comorbidities. This study sought to determine the prevalence of obesity and overweight among secondary school students whose parents which were mainly from the lower socioeconomic strata of society and whose health indices were largely unknown. The combined prevalence of obesity and overweight was $9.8 \%$ (1.0\% and $8.8 \%$ respectively), was not overly at variance with other studies from Nigeria [11]-[13], where the prevalence varied from rural to urban areas, and across various ethnic groups, rural youths being less obese than the urban. The prevalence of obesity and overweight was lower in these students compared to findings from South Africa [14]-[16], India [17], China [18], Saudi Arabia [19], [20], South America [21]-[24], Europe [25]-[27] and North America [28], [29]. This may be partly accounted for by the fact that these studies neither segregated public from private schools [17], [30] nor affluent [31] from the less endowed.

Girls were more obese/overweight than boys. This was very evident in the age range 15 to 17 years. This finding was corroborated by other workers [14]-[16], [20], [24], [26], [31]. This could be explained by the fact that the girls consumed more calories, being more involved in culinary chores and also were more sedentary in nature. However, some studies [21], [23], [29], [30] observed a preponderance of obesity and overweight in boys. This was attributed to dietary pattern, reduced outdoor activity rather than any genetic predisposition.

Being underweight was a feature found exclusively among the younger girls and a near equal mix of older and younger boys. So increasing age was not tantamount to being overweight. However, observations from South American children [21], [23] showed younger children having a higher proportion of obesity/overweight than the older, and elsewhere [16], [26] there being no gender difference in obesity prevalence among the younger children.

Dietary intake was not significantly associated with obesity in this study. The obvious limitation was that the authors could not assess the direct quantities of the foods consumed and thereby estimate the caloric values. The preference for calorie dense snacks and sweetened drinks is a known risk factor for obesity and overweight as noted by other studies [12], [19], [20], [21], [30].

Like diet, socio-economic level had no significant association with obesity/overweight in these students. This was probably because the population was more or less homogenous with a loose classification into the social classes. This finding was consistent with that of Ben-Bassey et al in Lagos, Nigeria [13]. In high income countries, food insecurity accompanies obesity and overweight in children as poorer persons tend to go for cheaper, energy-dense processed foods [28], [30], [32]. However, some other studies opine that the children of the more affluent tend to be more obese [15], [24], [27] being availed of a variety of foods in large quantities. There may be other additional factors like increased sedentary living. In many low and middle income countries low socioeconomic status is rather associated with the opposite - a decrease in obesity and an increase in stunting and undernutrition [18].

In this sudy obesity/overweight had an inverse relationship with physical activity. This is an established fact also observed in other studies [12], [20], [31], [33]. Physical activity underlies the body's energy expenditure and strikes a balance between calories consumed and those stored as body fat (resulting in obesity). The more vigorous and prolonged the physical activity, the more the energy utilized and the less the adiposity. Boys were more physically active than girls and this resulted in their being less obese/overweight.

Comparing the different anthropometric indices, the BMI performed better in identifying the students who were obese/overweight. WC and WHtR had a high specificity but low sensitivity. Inspite of its utility in classifying nutritional status in children, the ability of the BMI to predict body fat is contentious, as a high score may be from high levels of adiposity or from fat-free tissues [34], [35]. WC and WHtR have been advocated for screening for adiposity, due to the relationship between waist measurement and visceral adiposity [36], [37], which in itself is a better reflection of cardiometabolic risk factors. WC varies with different ethnicities [38], [39]. Another limitation of this study was the use of WC percentile charts of Canadian children as standard for comparism instead of African Black children, due to unavailability of requisite data.

\section{CONCLUSION}

Our findings indicate that a significant proportion of adolescents, especially older females, in Nnewi public secondary schools are obese, and buttress the importance of physical exercises in curbing adolescent obesity. In addition, the utility of WC and WHtR in identifying adolescents with obesity is highlighted. Although easier to perform and very specific, WC and WHtR seemed to miss many adolescents with obesity when compared to BMI. Therefore, a combination of these indices is recommended while screening adolescents for obesity.

Efforts should be intensified at creating awareness on the 
prevention and dangers of adolescent obesity. Routine assessment and interpretation of obesity indices should be fully integrated into the school health programmes to ensure prompt identification of obesity and early institution of remedial interventions.

\section{CONFLICTS OF INTEREST}

The authors declare that they have no conflict of interest.

\section{DISCLOSURE}

This research did not receive any specific grant from funding agencies in the public, commercial, or not-for-profit sectors.

\section{ETHICAL APPROVAL AND CONSENT}

Ethical approval was obtained from the institutional ethical committee of Nnamdi Azikiwe University Teaching Hospital. Permission to carry out the study was sought for and obtained from the Zonal Directorate of Post Primary School Service Commission and Anambra State Universal Basic Education Board, Nnewi Zone. Consent of participating school principals, parents/guardians, tutorial staff and students were duly obtained.

\section{ACKNOWLEDGEMENT}

We would like to thank all the school principals, tutorial staff and the students of the schools that participated in the study. We also express our sincere gratitude to the authorities of the Zonal Directorate of Post Primary School Service Commission and Anambra State Universal Basic Education Board, Nnewi Zone for permitting data collection.

\section{REFERENCES}

[1] World Health Organization. Report of the Commission on Ending Childhood Obesity. WHO 2016.

[2] Falkner B, Gidding SS, Ramirez-Garnica G, Wiltrout SA, West D, Rappaport EB. The relationship of body mass index and blood pressure in primary care pediatric patients. J Pediatr 2006; 148(2): $195-200$.

[3] Sorof JM, Lai D, Turner J, Poffenbarger T, Portman RJ. Overweight, Ethnicity, and the Prevalence of Hypertension in School-Aged Children. Pediatrics 2004; 113 (3): 475 - 482. Doi: 10.1542/peds.113.3.475

[4] Riley M, Bluhm B. High blood pressure in children and adolescents. American Family Physician 2012; 85 (7): 693 - 700.

[5] Ugochukwu EF, Onubogu CU, Ofora VC, Okeke KN, Uju CM. Blood pressure profiles and determinants of hypertension among public secondary school students in Nnewi, Southeast Nigeria. European Journal of Medical and Health Sciences 2020; 2(3). DOI: https://doi.org/10.24018/ejmed.2020.2.3.298.

[6] World Health Organization. Global Recommendations on Physical Activity for Health. WHO 2010.

[7] World Health Organization. Waist Circumference and Waist-Hip Ratio. Report of a WHO Expert Consultation. Geneva, 8-11 December 2008. World Health Organization 2011.

[8] WHO BMI-for-age and sex: percentile and z-score charts (5-19 years). Available at https://www.who.int>growthref; https://www.who.int>standards>

[9] Children's Hospital Research Institute of Manitoba, Canada: Waist:Height Ratio percentile charts for Boys and Girls 5-19 years and Waist percentile charts for Boys \& Girls 5-19 years. Available at: https://cpeg-gcep.net>upload.pdf; https://cpeg-gcep.net>bookfiles

[10] Oyedeji GA. Socio-economic and cultural background of hospitalized children in Ilesha. Nigerian Journal of Paediatrics 1985; 12: 111 117.

[11] Oduwole AA, Ladapo TA, Fajolu IB, Ekure EN, Adeniyi OF. Obesity and elevated blood pressure among adolescents in Lagos, Nigeria: a cross-sectional study. BMC Public Health 2012; 12: 616 http://www.biomedcentral.com/1471-2458/12/616. Doi 10.1186/1471-2458-12-616.

[12] Omuemu VO, Omuemu CE. The prevalence of overweight and its risk factors among adolescents in an urban city in Edo State. Niger J Clin Pract 2010; 13 (2): 128 - 133. PMID: 20499742.

[13] Ben-Bassey UP, Oduwole AO, Ogundipe OO. Prevalence of overweight and obesity in Eti-Osa LGA, Lagos, Nigeria. Obes Rev 2007; 8 (6): 475 - 479. https://doi.org/10.1111/j.1467 789X.2007.00408.x

[14] Kimani-Murage EW, Kahn K, Pettifor JM, Tollman SM, Dunger DB, Gomez-Olive XF, et al. The prevalence of stunting, overweight and obesity, and metabolic disease risk in rural South African children. BMC Public Health 2010; 10: 158 - 70 http://www.biomedcentral.com/1471-2458/10/158.

[15] Kimani-Murage EW, Kahn K, Pettifor JM, Tollman SM, KlipsteinGrobusch K, Norris SA. Predictors of adolescent weight status and central obesity in rural South Africa. Public Health Nutr 2011; 14 (6): 1114 - 1122. Doi: 10.1017/S1368980011000139.

[16] Otitoola O, Oldewage-Theron W, Egal A. Prevalence of overweight and obesity among selected schoolchildren and adolescents in Cofimvaba, South Africa. South African Journal of Clinical Nutrition 2020. Doi:10.1080/16070658.2020.1733305. Available at https://doi.org/10.1080/16070658.2020.1733305

[17] Stigler MH, Arora M, Dhavan M, Shrivastav R, Reddy KS, Perry CL. Weight-related concerns and weight-control behaviors among overweight adolescents in Delhi, India: A cross-sectional study. International Journal of Behavioral Nutrition and Physical Activity 2011; 8: 9. http://www.ijbnpa.org/content/8/1/9. Doi: 10.1186/1479 5868-8-9.

[18] Ip P, Ho FKW, So H-K, Chan DF-y, Ho M, Tso W, et al. Socioeconomic gradient in childhood obesity and hypertension: A multilevel population-based study in a Chinese community. PLoS ONE 2016; 11(6): e0156945. Doi: 10.1371/journal.pone.0156945.

[19] Al-Rethaiaa AS, Fahmy AA, Al-Shwaiyat NM. Obesity and eating habits among college students in Saudi Arabia: a cross sectional study. Nutrition Journal 2010; 9: $39-48$ http://www.nutritionj.com/content/9/1/39.

[20] Mahfouz AA, Shatoor AS, Khan MY, Daffalia AA, Mustafa OA, Hassanein MA. Nutrition, physical activity, and gender risks for adolescent obesity in Southwestern Saudi Arabia. Saudi J Gastroenterol 2011; 17: 318 - 22. www.saudijgastro.com. PubMed ID: 21912058 . Doi: 10.4103/1319-3767.84486.

[21] Duncan S, Duncan EK, Fernandes RA, Buonani C, Bastos KD-N, Segatto AFM et al. Modifiable risk factors for overweight and obesity in children and adolescents from Sao Paulo, Brazil. BMC Public Health 2011; 11: 585 - 93. http://www.biomedcentral.com/1471 2458/11/585.

[22] Silva DAS, Pelegrini A, de Lima e Silva JMF, Petroski EL. Epidemiology of abdominal obesity among adolescents from a Brazilian State. J Korean Med Sci 2011; 26: 78 - 84. http://jkms.org. doi: 10.3346/jkms.2011.26.1.78

[23] Garza JR, Perez EA, Prelip M, McCarthy WJ, Feldman JM, Canino $\mathrm{G}$, et al. Occurrence and correlates of overweight and obesity among island Puerto Rican youth. Ethn Dis 2011; 21 (2): 163 - 169.

[24] Guedes DP, Rocha GD, Silva AJRM, Carvalhai IM, Coelho EM Effects of social and environmental determinants on overweight and obesity among Brazilian schoolchildren from a developing region. Rev Panam Salud Publica 2011; 30 (4): 295 - 302.

[25] Athanasopoulos DT, Garopoulou AI, Dragoumanos VP. Childhood obesity and associated factors in a rural Greek island. Rural and Remote Health 2011; 11: 1641. Available: http://www.rrh.org.au.

[26] Yuca SA, Yilmaz C, Cesur Y, Dogan M, Kaya A, Basaranoglu M. Prevalence of overweight and obesity in children and adolescents in Eastern Turkey. J Clin Res Pediatr Endocrinol 2010; 2 (4): 159 - 163. Doi: 10.4274/jcrpe.v2i4.159. PMID: 21274316.

[27] Bereket A, Atay Z. Current status of childhood obesity and its associated morbidities in Turkey. J Clin Res Pediatr Endocrinol 2012 4 (1): 1 - 7. Doi: 10.4274/jcrpe.506. PMID: 22394698

[28] Eagle TF, Sheetz A, Gurm R, Woodward AC, Kline-Rogers E, Leibowitz R, et al. Understanding childhood obesity in America. Linkages between household income, community resources, and children's behaviors. Am Heart J 2012; 163 (5): 836 - 843

[29] Isasi C, Whiffen A, Campbell E, Florez Y, Freeman K, Wylie-Rosett J. High prevalence of obesity among inner-city adolescent boys in the Bronx, New York: Forgetting our boys. Preventing Chronic Disease 2011; 8 (1). http://www.cdc.gov/pcd/issues/2011/jan/10_0009.htm. Accessed May 23, 2020.

[30] Oh I-H, Cho Y, Park S-Y, Oh C, Choe B-K, Choi J-M, et al Relationship between socioeconomic variables and obesity in Korean 
$\begin{array}{llllllll}\text { adolescents. } \quad J & \text { Epidemiol } & 2011 ; & 21 & \text { (4): } & 263 & - & \end{array}$ Doi:10.2188/jea.JE20100099

[31] Jain S, Pant B, Chopra H, Tiwari R. Obesity among adolescents of affluent public schools in Meerut. Indian Journal of Public Health 2010; 54 (3). www.ijph.in. doi: 10.4103/0019-557X.75740.

[32] Morgen CS, Mortensen LH, Rasmussen M, Andersen AN, Sorensen TIA, Due P. Parental socioeconomic position and development of overweight in adolescence: longitudinal study of Danish adolescents. BMC Public Health 2010; 10: 520 - 30 http://www.biomedcentral.com/1471-2458/10/520.

[33] Perez A, Hoelscher DM, Springer AE, Brown HS, Barroso CS, Kelder SH, et al. Physical activity, watching television, and the risk of obesity in students, Texas, 2004-2005. Preventing Chronic Disease 2011; 8 (8). http://www.cdc.gov/pcd/issues/2011/may/10_0007.htm. Accessed May 23, 2020.

[34] Khoury M, Manlhiot C, McCrindle BW. Role of the Waist/Height Ratio in the cardiometabolic risk assessment of children classified by body mass index. Journal of American College of Cardiology 2013; 62 (8): 742 - 51. http://dx.doi.org/10.1016/j.jacc.2013.01.026 .

[35] Jensen NS, CamargoTdeF, Bergamaschi DP. Body mass index and waist circumference are good indicators for classifying children's nutritional status. Ciencia \& Saude Coletiva 2016; 21 (4): 1175 1180. Doi: 10.1590/1413-81232015214.138712015. PMID: 27076016

[36] Li C, Ford ES, Mokdad AH, Cook S. Recent trends in wais circumference and waist-height ratio among US children and adolescents. Paediatrics 2006; 118(5): e1390 - e1398. www.pediatrics.org/cgi/doi/10.1542/peds.2006-1062.

[37] Savva SC, Tornartis M, Savva ME, Kourides Y, Panagi A, Silikiotou $\mathrm{N}$, et al. Waist circumference and waist-to-height ratio are better predictors of cardiovascular disease risk factors in children than body mass index. International Journal of Obesity 2000; 24: $1453-1458$ www.nature.com/ijo.

[38] Schwandt P, Haas G-M. Waist circumference in children and adolescents from different ethnicities. Childhood Obesity, Dr. Sevil Ari Yuca (Ed.) 2012. ISBN: 978-953-51-0374-5, In Tech, Available from: http://www.intechopen.com/books/childhood-obesity/waistcircumference-in-children-and-adolescents-from-different-ethnicities.

[39] Bacopoulou F, Efthymiou V, Landis G, Rentoumis A, Chrousos GP Waist circumference, waist-to-hip ratio and waist-to-height ratio reference percentiles for abdominal obesity among Greek adolescents. BMC Pediatrics 2015; 15: 50. Doi: 10.1186/s12887-015-0366-z.

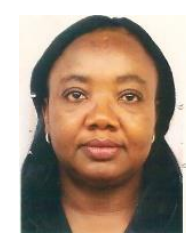

Dr. Ebelechuku Francesca Ugochukwu

Professor of Paediatrics and Chief Consultant

Paediatrician

MB.BS, FWACP (Paediatrics)

Past Head of Paediatrics and Past Dean, Faculty of Medicine.

Dept of Paediatrics, Nnamdi Azikiwe University,

Awka, Nigeria.

Email: ef.ugochukwu@unizik.edu.ng

Special interest in Paediatric Infectious Diseases (HIV \& TB), Adolescent Medicine, Paediatric Nutrition \& Neonatology.

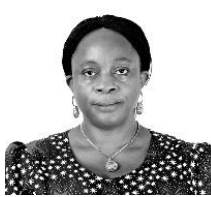

Dr.Chinyere Ukamaka Onubogu MB. BS, FWACP (Paediatrics). Consultant Paediatrician and Lecturer at Dept of Paediatrics, Nnamdi Azikiwe University, Awka.

Email: cu.onubogu@unizik.edu.ng

Author of many peer reviewed publications. Special interest in Paediatric Infectious Diseases (TB \& HIV), Paediatric Nutrition and Adolescent Health.

Dr. Kenneth N. Okeke, MB. BS, FMCPaed

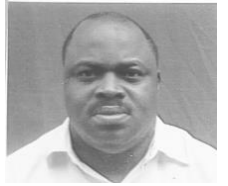

Azikiwe University, Awka.

Email:kn.okeke@unizik.edu.ng.

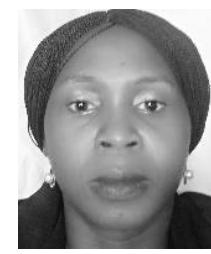

Dr. Victoria C. Ofora. MB. BS, FWACP (Paediatrics). Post Fellowship Semior Registrar at Dept of Paediatrics, Nnamdi Azikiwe University Teaching Hospital, Nnewi.

Email: victoriaofora1@gmail.com

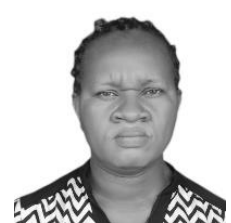

Dr. Chinenye MaryQueen Uju, MB. BS.

Senior Registrar in Paediatrics at Nnamdi Azikiwe University Teaching Hospital, Nnewi.

Email: ujuisidore@yahoo.co.uk 